\title{
EXPERIENCIA CLÍNICA, PARTE I Una aproximación a un caso práctico de comunicación terapéutica
}

\author{
Clinical Experience, part I
}

An approach to a practical case of therapeutic communication.

\section{Experiência clínica, parte I}

Uma aproximação com um caso prático de comunicação terapêutica.

Isabel Huércanos Esparza

DUE por la Universidad Pública de Navarra, Licenciada en Enfermería Europea por University of Brighton (UK)

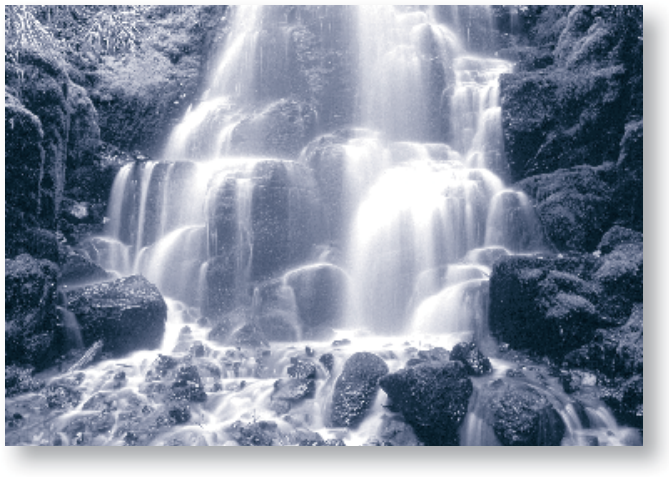

CLINICAL EXPERIENCE, PART I

An approach to a practical case of therapeutic communication.

\section{ABSTRACT}

Nursing is an eminently humanistic career, based on therapeutic communication. It requires an especial attitude, sensitivity, respect and love for human beings, it's in this context where we should work as a nurses. This intervention is based on the belief that to do our work therapeutic and significant to our patients, it must take into account which is the meaning of the disease for each person and comforted him or her in an active process to find together new behaviours more constructives for the person.
This work is a real theoric and practice case, what it happened in a hospital, specifically in a Cardiology Unit, with a woman who had a myocardial infarction. We can find in this conversation deep emotions and feelings, she has taken stock of the events related to the $\mathrm{MI}$ and she consider her future after it.

The work has two communicative interventions, first of them is developed in this article, we analize patient's fears and its implications. In the second part, we discuss more subjects, the aims were to know if fears were resolved, strategies were put on practice, evaluate her self-esteem and training her to become self-sufficient for her own care.

Keywords: Therapeutic relationship, nursing, communicative intervention.

\section{EXPERIÊNCIA CLÍNICA, PARTE I}

Uma aproximação com um caso prático de comunicação terapêutica.

\section{RESUMO}

A enfermagem é uma profissão, eminentemente, humanística e voltada para a relação de ajuda. Tal fato requer uma atitude de especial sensibilidade, respeito e amor perante o cui- 
dado do ser humano, pois é nessa perspectiva que se deve situar o trabalho da enfermagem. Assim, a presente investigação volta-se para a crença de que o trabalho da enfermagem deve contribuir para um resultado terapêutico e significativo, levando-se em consideração o significado do adoecimento para a pessoa e o acompanhamento dela em um processo ativo com o intuito de encontrar juntos novas maneiras ou formas de conduta mais construtivas na interação com ela (Piulach, 1984).

O presente trabalho trata de um caso teórico-prático, ocorrido em uma unidade de atendimento em cardiologia, no qual uma senhora havia sofrido um IAM. Nessa situação, colocase em jogo emoções e sentimentos profundos, bem como se descreve o ocorrido e se planeja o futuro depois do agravo.

Incluem-se duas intervenções comunicativas: sendo a primeira apresentada neste artigo, analisando-se o medo que a pessoa demonstrou e o que comportava tal situação. Na segunda (que se apresenta na parte II do artigo), são enunciados outros temas, com o intuito de avaliar se o sentimento de medo resolvido, e se foram colocadas em prática estratégias e se foi valorizada a autonomia da pessoa, além de se buscar a capacitação para o seu auto cuidado.

Palavras chave: Relação de ajuda, Enfermagem, intervenção comunicativa.

\section{RESUMEN}

Enfermería es una profesión eminentemente humanística y de relación de ayuda, que requiere una actitud de especial sensibilidad, respeto y amor frente al ser humano y es en este contexto en el que debe situarse nuestro quehacer enfermero. Esta intervención se basa en la creencia de que para que nuestra labor como enfermeras/os resulte terapéutica y sig- nificativa debe tener en cuenta el significado de la enfermedad para la persona y acompañarla hacia un proceso activo con el fin de encontrar juntos nuevas conductas más constructivas para ella (Piulach, 1984).

Este trabajo es un caso teórico-práctico real en una unidad de hospitalización de cardiología, con una mujer que ha sufrido un IAM. En él se han puesto en juego emociones y sentimientos profundos, se hace balance de lo ocurrido y se plantea el futuro tras el acontecimiento de enfermedad.

Incluye dos intervenciones comunicativas, la primera se presenta en este artículo, se analiza el miedo que ella muestra y lo que conlleva. En la segunda (se presenta en la parte II del artículo), se tratan más temas, con el propósito de evaluar si el miedo se ha resuelto, si se han puesto en práctica estrategias y valorar su autoestima, además de buscar la capacitación para su autocuidado.

Palabras clave: Relación de ayuda, enfermería, intervención comunicativa.

\section{ANÁLISIS DEL PRIMER ENCUENTRO}

María, es una mujer de 55 años. Se encuentra en una situación de crisis, ha sufrido un infarto de miocardio y se encuentra en el $5^{\circ}$ día de hospitalización.

En el momento de nuestro primer encuentro, no la conocía, ni tampoco conocía su diagnóstico. Entro en su habitación porque su enfermera me pide que le quite una vía central. Por lo que esta primera intervención comunicativa se produce de manera improvisada.

$\mathrm{Al}$ entrar saludo a las dos pacientes por su nombre y me dirijo a la cama de María, ella me saluda con aparente bienestar, le digo que vengo a quitarle la vía, ella extiende el brazo, le quito los apósitos con delicadeza y empiezo 
a extraerla. Ella se queja de dolor y yo le digo que lo haré despacio y cuando ella quiera pararé un rato, lo hacemos así hasta que está fuera, le pongo una gasa y un esparadrapo y le pido que apriete un poco. Entonces ella se lleva la mano al punto de punción y comienza a llorar intentando contener las lágrimas y girando la cara para que no la vea, en este momento comienza la interacción.

María había sufrido un infarto y hacía tres días había subido a planta tras pasar dos días en la UCI.

Es una mujer casada, madre de tres hijos, tiene amigos a los que ve de vez en cuando. Trabajaba de cuidadora a domicilio, durante varios años cuidó de la misma persona, hace tres meses murió esa persona y desde entonces se ha ocupado únicamente de las tareas del hogar.

Según Cibanal et al. (2003), constatamos que cuando las palabras no han sido dichas, aparece como un deterioro en el cliente, cuando no comunicamos lo que sentimos, vivimos, pensamos,... resulta que nos sentimos como aislados y nuestra ansiedad, miedo o angustia aumenta. Es por esto que creo que María, lanzó una llamada de atención al llorar, porque necesitaba expresar esos sentimientos que la recorrían tras la vivencia del infarto.

Ella enseguida expresa que está mal, por lo que al percibir que necesita hablar cojo una silla y me siento en ella junto a la cama de María, a su lado, teniendo en cuenta que las actitudes no verbales tienen una gran importancia en la comunicación (Cibanal, Arce y Carballal, 2003), lo hago para estar a la misma altura y poder hablar de tú a tú, mirándola de frente, con mi cuerpo ligeramente inclinado hacia ella en actitud de escucha. He estado atenta a no cruzar los brazos para que no pensase que estaba a la defensiva.
Con el fin de demostrarle acogida, interés y cordialidad he mantenido el contacto visual con ella, ya que, mediante la mirada el cliente puede percibir que nos esforzamos para acercarnos psicológicamente a él, incluso cuando ella no me miraba la he seguido mirando, he procurado que mi rostro mostrase comprensión y calma, acompañado de una sonrisa en ciertos momentos y asintiendo a modo de feedback, para darle a entender mi entendimiento y aceptación (Cibanal et al. 2003).

Al principio de la interacción, tengo la necesidad de informarme de su situación médica para poder darle respuestas apropiadas y poder comprenderla mejor, por eso le pregunto qué le han dicho los médicos.

Al contarme que los médicos la han informado de que ha tenido un pequeño infarto, me intereso por como está viviendo ella eso y le pregunto “¿y cómo te hace sentir eso?” ella expresa su malestar pero al principio no encuentra palabras para expresarlo, yo espero un poco manteniéndome en silencio y mirándola, a continuación le pregunto “qqué es lo que te hace sentir tan mal?”. Entonces ella me cuenta que lo que le crea ese malestar es no saber qué va a sucederle después. En éste punto me adelanto un poco preguntándole si se refiere a cuando se vaya de alta. Me doy cuenta de que hubiese sido mejor una pregunta del tipo ia qué te refieres con después? Para que fuese ella misma la que me diga lo que la preocupa. De todas formas, ella afirma que se refería a eso y me cuenta la vivencia del dolor que sintió y su preocupación por no saber reconocerlo si se volviese a producir diciendo "...cuando me vaya a casa, ¿voy a estar todo el tiempo en que es eso cada vez que tenga un dolor?!", al decirlo los músculos de su cara se tensan y su tono de voz se vuelve agresivo en cierto modo, esto denota poca confianza en sí misma (Cibanal et 


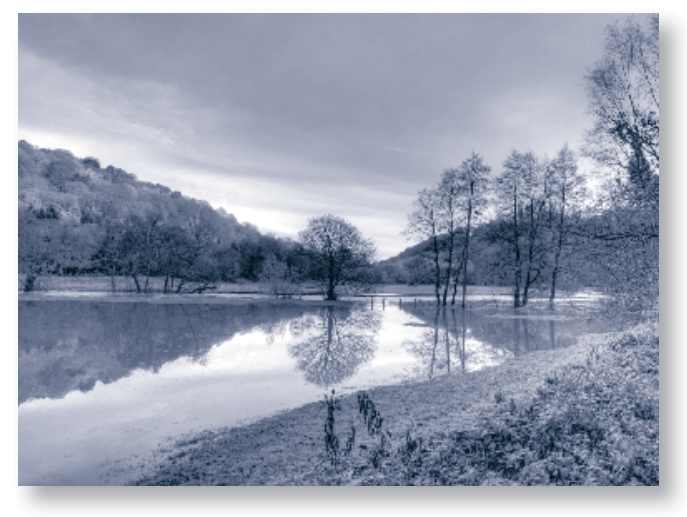

al., 2003) y me hace pensar que es algo que le preocupa mucho.

Por eso, le digo "entiendo que esa incertidumbre te haga sentir mal", con el fin de ofrecerle presencia, para que se sienta arropada y acompañada, y mostrando una actitud empática en la que ella pueda percibir que estoy entrando en su mundo personal (Giordani, 1997). Además le digo "mientras estás aquí nos puedes llamar al timbre siempre que tengas algún dolor y con un ECG sabemos si es eso o no, y podemos evitarlo con un spray" esto lo hago para que se sienta protegida y acompañada, que es otro de los aspectos que ayudan a resolver el miedo (Basurto, 2006-2007). A continuación, le proporciono información acerca de eso que le inquieta tanto, explicándole cómo puede reconocer el dolor, cómo actuar en caso de que se produzca y comentándole que apenas tiene efectos secundarios. Con la intención de que se sienta más segura y el miedo se reduzca mediante la información "self-service" (Basurto, 2006-2007).

Parece que la respuesta que le doy la tranquiliza, porque se muestra menos tensa $y$ sonríe, me intereso por si hay algo más que la preocupe. Entonces ella dice "...tampoco quiero estar llorando con mis hijos y con todos", esta frase me sugiere que no se permite a ella misma que afloren señales y sentimientos que indican la insatisfacción que siente por necesidades no cubiertas (Basurto, 2006-2007), como la de seguridad y quizás la de autoestima. Con el fin de que se puedan satisfacer, sigo preguntándole para averiguar si tiene posibilidad dentro de su círculo familiar de drenar emociones "¿has hablado con ellos sobre cómo te estás sintiendo después de lo que te ha pasado?", ella expresa "con mis hijos no he hablado, no quiero hacerlos sufrir". Al analizar la interacción me doy cuenta de que esta frase puede indicar que María es una mujer acostumbrada a cuidar de su familia, pero no sé hasta que punto es consciente de que ella también es importante. Un poco después, dice que no quiere que la gente que viene a verla y que no pertenece a su familia más cercana le vea mal y reconoce que delante de ellos intenta aparentar que se siente bien. En este momento, podría haber explorado su autoestima, para que en caso de que no se valore demasiado a ella misma, intentar que aprenda que ella también es una persona valiosa e importante.

Sin embargo, lo que hago es preguntarle "¿no crees que a ellos les gustaría saber cómo te sientes ahora?", con el fin de detectar si puede hablar sobre cómo se está sintiendo con sus hijos también, puesto que para tratar el miedo es importante hablar sobre él, explicitarlo (Basurto, 2006-2007), pero ella insiste en que no quiere y entonces le preguntó por otras personas-recurso que ella pueda tener. Entonces, ella me habla de su marido y su hermana como esas personas, incluso refiere de nuevo que su hermana ha pasado también por un infarto y es la que mejor la entiende, por lo que con esto ella ya ha podido empezar a plantearse hablar sobre sus emociones con personas de su entorno. Entonces con una reformulación, de tipo reiteración: "entonces, tienes a tu marido y a tu hermana..." busco que ella perciba que la escucho con atención e interés y con la intención de 
que tome conciencia de su situación y lo que me ha expresado de manera que pueda poner en marcha acciones para la resolución de ese miedo (Cibanal et al., 2003). Parece resultar porque tras esto comenta: "Sí, además mi marido va a venir después de comer y hablaré con él cuando venga sí..." Aquí percibo que se ha producido un cambio, María ahora es consciente de que hablar sobre sus sentimientos y vivencias en este momento y compartirlo puede ser beneficioso para ella y reducir la carga que tiene. Finalmente María verbaliza claramente el miedo, al haber favorecido que ella pudiese hablar conmigo de cómo le hace sentir esta situación, haciéndole preguntas abiertas y empleando la reformulación: “...me da miedo no salir de ésta..." ya me había hecho pensar en la presencia de miedo con frases anteriores como "lo que me preocupa es no saber qué va a pasar" "cuando me duele un poco la espalda por la postura o algo así lo primero que pienso es si será otra vez el infarto..."

He valorado si ese miedo es real, esto me interesa porque es el primer paso para poder ayudarla, creo que lo es en cierta medida porque aunque bien es cierto que no tiene porque volver a darse un infarto ya le ha sucedido una vez.

Según Maslow (1991) "el organismo está dominado por las necesidades insatisfechas al igual que la organización de su comportamiento", en este caso el miedo que experimenta María es una expresión de que su necesidad de seguridad no está satisfecha, como nombra más adelante el autor, la necesidad de seguridad abarca necesidad de estabilidad, protección, ausencia de miedo y ansiedad, etc. Pensando en esto y en la situación en la que encontré a María, me plantee en qué manera ese miedo la afectaba observando que en cierta forma la inmoviliza, ya que, como ella expresa no se

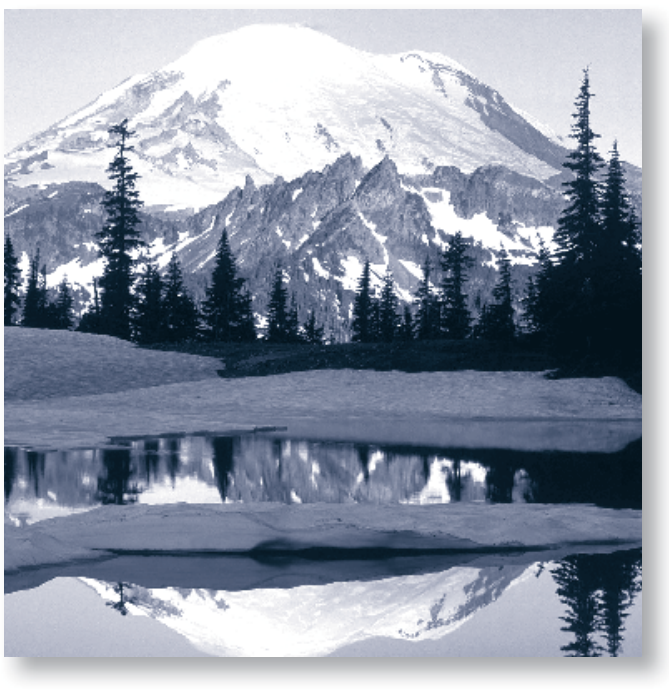

siente con ánimos para levantarse de la cama. Considero que si este miedo no se trata, la puede inmovilizar incluso en otros aspectos de su vida una vez se vaya de alta.

Una vez detectado el miedo y haberle dado dimensión (Basurto, 2006-2007), juntas hemos ido analizando que puede hacer, buscando que ella sea consciente de que está en su mano evitar que se repita el infarto y así se sienta más segura. Para ello, quiero detectar qué es lo que a ella le supone mayor dificultad y con qué relaciona el infarto.

Hemos hablado sobre cambios que están en sus manos para reducir ese miedo, en ese momento le digo "pero no tiene porqué volver a pasar, con los cambios en los estilos de vida, es decir, en la dieta, el ejercicio... hay muchos estudios que demuestran que el riesgo se reduce muchísimo"

He llegado a ayudarle para que saque la clave del miedo, la imposibilidad que ella ve para dejar de fumar cuando dice "... lo que me va a costar es dejar de fumar, eso sí que me va a costar..." esto me hace pensar que hace una relación directa entre el tabaco y el infarto y es esa imposibilidad que cree que tiene para dejar de fumar lo que la hace pensar que se repetirá. 
Aunque muestra su intención de abandonarlo "pero se acabó, ya veo que no se puede y no se puede", le refuerzo positivamente diciéndola "entiendo que tiene que ser duro(empatía) pero esa disposición que tienes para dejar de fumar $y$ ese ánimo es lo más importante" buscando que se sienta apoyada y le dé ánimo para el cambio, ya que es necesario que el terapeuta acompañe el camino que el cliente ha asumido libremente con intervenciones reforzantes y sostenedoras del compromiso (Giordani, 1997), es por esto que empleo el refuerzo positivo en varias ocasiones en mis interacciones con María.

Ella misma en ese momento parece que se anima y sonriendo dice "además ya llevo sin fumar todo el tiempo que llevo aquí (en el hospital)..." le vuelvo a dar refuerzo positivo y le doy información, en ese momento con la intención de que ella supiese que realmente es una decisión importante que le puede ayudar mucho en su situación de salud y que la tranquilice, en relación a que ella puede actuar, pero al hacer el análisis me doy cuenta de que me adelanto y hubiese sido mejor explorar qué supone para ella llevar tanto tiempo fumando "por lo menos 20 " y que ella pudiese expresar sus dificultades y sus dudas, esto me hubiese permitido que la EpS que le doy esté más centrada en ella e individualizada.

Ella se sorprende de la información que le doy y sonríe, lo que me lleva a pensar que realmente le reconforta lo que le digo, aunque hubiese sido más apropiado más tarde.

Después le pregunto si le han entregado la guía del paciente coronario (es una guía que informa a los pacientes de los factores de riesgo del infarto, aunque no da información sobre cómo modificarlos), ella me responde que sí y que la ha leído, por lo que, la información que yo le doy refuerza y amplía esa que ya tenía.

Tras este pequeño paréntesis, vuelvo a pre- guntarle por sus apoyos, pienso que es más adecuado, una vez identificada su principal dificultad "con respecto a lo de dejar de fumar, ¿tienes gente que te apoye en casa?" con el fin de descubrir si en su casa se le va a dificultar el dejarlo o no, pero ninguno fuma y siempre han querido que lo deje.

Me habla de otro momento en el que lo intentó dejar y no pudo. Aquí me planteo al leer la conversación que hubiese sido bueno seguir hablando de la anterior vez que lo intentó para ver las diferencias entre la situación que vivía ella antes y la de ahora, con el fin de potenciar las diferencias que hay en este momento como su ánimo, concienciación, mayor información para que pueda ver que no tiene porque suceder lo mismo.

Sin embargo, en el momento de la interacción ella vuelve a decir que "tengo muy claro que se acabó", le doy refuerzo y protección de nuevo cuando le toco y le digo "el ánimo que tienes para dejarlo es lo más importante" y reconozco sus recursos: “...y teniendo el apoyo de tu familia yo creo que será más fácil", ya que parece que su familia está ahora más encima de ella para que lo deje tras el infarto.

Pensando sobre cómo habla de su familia como fundamental punto de apoyo para ella, me planteo intentar averiguar qué tipo de apoyo le dan realmente, sobretodo su marido que es con el que más tiempo pasa, para ver si su apoyo se basa en la escucha o puede además hacer algo por ella.

Siguiendo con el diálogo, yo le he ofrecido posibilidades que es posible que ella no conozca, como la consulta de la enfermera en el centro de salud o grupos de ayuda para la deshabituación tabáquica en la comunidad, además de un folleto para que pueda ir leyendo estos días de ingreso. Ella es una mujer con recursos y busca otra posibilidad y la propone: "Ahora 
que lo dices, cerca de mi casa hay una herboristería en la que tienen una persona para eso $y$ hacen lo de los grupos", había pensado hacerlo anteriormente pero finalmente no lo había hecho nunca.

Reconozco que es una buena forma de hacerlo y le comento que le traeré un folleto si quiere, ella se muestra dispuesta.

Le doy algunas estrategias que se destacan en la información que le entregaré por escrito y que le pueden ser de ayuda como "...por cada semana que pasas sin fumar hacerte un regalito, para animarte a seguir $y$ darte un premio por ese éxito", a ella parece gustarle la idea y sonríe proponiendo "con el dinero que me gastaba antes en tabaco me lo voy a ir guardando para irme a cenar un día con mi marido o al cine...", la vuelvo a reforzar diciéndole "me parece muy buena idea" y sigo "otra de las cosas que destacan es que la mayor dificultad es que el tabaco se asocia a determinados momentos...lo que se puede hacer es cambiar de hábitos las primeras semanas que es cuando más cuesta", ella muestra que lo ha entendido y vuelve a proponer tácticas que cree que le pueden ayudar con la información que le he dado y adaptándola a sus propios gustos “... en vez de tomarme un café después de comer me puedo tomar una manzanilla o un Colacao o nada ¿no?”, continúa "sí así creo que es más fácil. Tengo bien claro que no puedo volver a fumar, además si cojo un cigarro y me ven mis hijos me matan", esto último aunque en su momento no lo confronté con ella, ahora me lleva a pensar en qué medida sus hijos la pueden ayudar porque se me plantea la duda de si intentan que deje de fumar mediante "amenazas" o haciéndola sentir culpable, porque esa no sería la manera adecuada y tendría que planteárselo en el próximo encuentro.

Ya al final de la conversación, le ofrezco mi disponibilidad para continuar hablando y que me pueda plantear lo que necesite, haciéndole ver cual es mi función y trabajo con ella y cuánto durará (Basurto, 2006-2007): “estos días que vas a estar aquí podemos seguir hablando sobre esto y sobre los demás factores de riesgo que te puedan preocupar"

Continúo preguntándole cuando le van a hacer el cateterismo, ella contesta que cree que el martes. Con el fin de que me dé un feedback de lo que ha comprendido sobre la información que le han dado los médicos le pregunto “¿te han explicado lo que es?”, ella explica brevemente en qué consiste de forma correcta, le pregunto si sabe que es en el otro hospital principal de la ciudad y ella contesta que no se lo habían comentado, entonces le explico brevemente la organización en estos casos. Le doy esta pequeña información para evitar la ansiedad que esta prueba puede provocar en los pacientes y que como he citado antes está relacionado con la necesidad de seguridad de las personas. Aunque en realidad no he comprobado antes si este es su caso o si se siente tranquila con respecto al cateterismo.

Mientras hablamos sobre el cateterismo y ella me ha explicado en qué consiste dice "todo eso me lo sé porque a mi hermana ya se lo hicieron y le pusieron varios muellecitos de esos", entonces para que ella pueda pensar en su propio miedo y darse cuenta de que hay personas que han pasado por lo mismo y se encuentran bien, teniendo en cuenta expresiones de $\mathrm{Ma}$ ría durante nuestra charla "cuando estaba en la UCI y le pregunté a mi hermana ¿y ahora qué?" Y ella me dijo "ahora a vivir" o cuando me ha dicho que el dolor lo tuvo mientras estaba andando con su hermana, y me han llevado a pensar que su hermana estaba bien le pregunto “'y cómo está tú hermana?”, María responde "como nueva y se lo hicieron hace 8 años" 
Hice esa pregunta con la intención de que ella se diese cuenta de que es posible salir de esa situación y seguir con su vida.

Termina nuestra conversación y ella me dice "me he quedado más tranquila después de hablar contigo"..."ya estoy mejor", por lo que parece que le he ayudado a que el miedo que sentía se reduzca y ha empezado a pensar en estrategias para ese cambio que al principio de nuestro encuentro veía muy difícil, la ha desbloqueado en cierta medida porque dice “... después me levantaré un poco"

Me presento porque todavía no lo había hecho puesto que la interacción surgió de improviso y nos despedimos tras recordarle mi disponibilidad, con la intención de que me vea también como un recurso para ella (Basurto, 2006-2007) y me comprometo a acompañarle en su decisión de dejar de fumar.

\section{CONCLUSIONES}

La teoría habla de varios aspectos a tratar para la gestión del miedo que actúan de antídotos (Basurto, 2006-2007), entre ellos dar la información que el paciente necesite y no la que como profesionales consideramos que debe tener, ya que, la relación de ayuda no es un proceso unidireccional en el que la persona prodiga la ayuda a un cliente que la recibe (Chalifour, 1994). Por ello, se da prioridad a los temas que a ella más le preocupaban, como son el dolor torácico y la deshabituación tabáquica, teniendo en cuenta una de las principales ideas de Isabel Serrano (1997), la importancia de que nuestro mensaje sea significativo para la persona, aquello que "conecte con la vivencia actual de los problemas de salud, con su trayectoria cultural, con sus experiencias de enfermedad. Por lo que es necesario tener como punto de partida la experiencia de las personas con las que trabajamos". Aunque en otros mo-

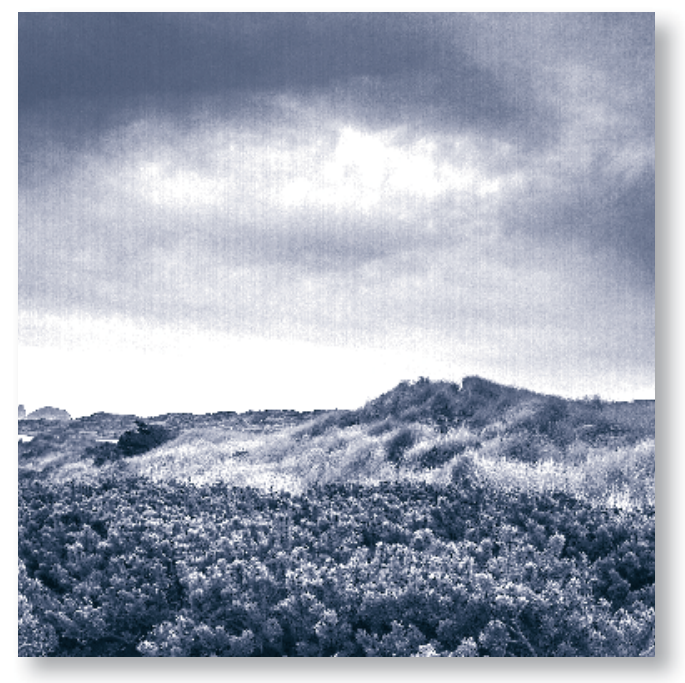

mentos me planteo abordar otros temas con María, para valorar qué necesita para afrontar esta situación y su futuro autocuidado una vez esté en su hogar.

Dado que la información se debe dar de manera dosificada, tal y como dice De Lorenzo (2005-2006) porque el paciente sólo puede retener unos puntos clave, por ello, planifico repartirla en los diferentes encuentros que tengamos de acuerdo a las necesidades que ella plantee.

En cuanto al miedo, se ha observado si es real, le hemos dado dimensión y hemos visto qué es lo que ella puede hacer para resolverlo (Basurto, 2006-2007)

Otro de los aspectos a analizar es la experiencia previsible, en este caso es posible que el infarto se vuelva a dar si no se trabaja para evitarlo. María ha mostrado su interés y compromiso para el cambio, ha decidido la meta, además de líneas de acción para lograrlas (Marroquín, 1991) y yo he procurado articular la teoría en la práctica demostrándole presencia y escucha activa. Además de protección, apoyo, disponibilidad, contacto congruente con el mensaje verbal (Cibanal et al., 2003) pretendiendo con esto que María se sintiese 
importante, escuchada, apoyada, que pudiese expresar su vivencia, sus dificultades, preocupaciones y miedos en relación con ellas. Que pudiese expresar y afrontar ese miedo y que se sintiese capaz de iniciar cambios, valorando el pasado y planificando el futuro.

He estado atenta a analizar su comportamiento no verbal, aunque en ocasiones me he despistado de esto intentando pensar la respuesta más apropiada o la pregunta oportuna.

\section{BIBLIOGRAFÍA}

- Basurto, S. (2006-2007) Cuidados enfermeros. Bibliografía no publicada para la asignatura Cuidados Holistas. Material del Aula. Escuela de Enfermería de Osakidetza Vitoria

- Chalifour, J. (1994). La relación de ayuda en enfermería. Una perspectiva holística-humanística. Barcelona: SG Editores.

- Cibanal, L, M.C Arce y M.C. Carballal (2003). Técnicas de comunicación y relación de ayuda en Ciencias de la salud. Madrid: Elsevier.

- De Lorenzo, E. (2005-2006). Educación para la salud. Bibliografía no publicada. Escuela de Enfermería de Osakidetza, Vitoria-Gasteiz

- Giordani, B. (1997). La relación de ayuda: de Rogers a Carkhuff. Bilbao: Desclée De Browuer

- Marroquín, M. (1991). La relación de ayuda en Robert R. Carkhuff. Bilbao: Mensajero.

- Maslow, A.H. (1991). Motivación y personalidad. Madrid: Díaz de Santos.

- Piulachs, M.T. (1984). Naturaleza de la atención de enfermería y su posición en el contexto general de los cuidados de salud. Nursing, Enero- Febrero: 19-27.

- Serrano, I. (1997). La Educación para la salud del siglo XXI. Comunicación y salud. Madrid: Díaz de Santos 